\title{
On the Capacity Region of the Gaussian Z-channel
}

\author{
Nan Liu Sennur Ulukus \\ Department of Electrical and Computer Engineering \\ University of Maryland, College Park, MD 20742 \\ nkancy@eng.umd.edu_ulukus@eng.umd.edu
}

\begin{abstract}
We investigate the capacity region of the Gaussian Z-channel with a small crossover link gain, i.e., $\alpha \leq 1$. For the case of $\alpha<1$, we provide an achievable region, and the converse for most of the achievable region. We also derive lower and upper bounds for the part of the region where the capacity boundary is unclear. For the case of $\alpha=1$, we determine the capacity region exactly.
\end{abstract}

\section{INTRODUCTION}

With recent advances in the theory, technology and applications of wireless communications, the network structures of interest are moving away from traditional networks such as the multiple access channel (MAC) and the broadcast channel (BC) which model cellular systems, and towards arbitrary network structures which model ad-hoc wireless networks. One important such network is the interference channel [1] [6]. An interference channel is a simple two-transmitter tworeceiver network, where each transmitter has a message for only one of the receivers. A more general network structure is the X-channel [7], where the channel is the same as the interference channel except that both transmitters have messages for both receivers. Unfortunately, most problems in network information theory, including the simple interference channel, have been open for quite a number of years.

Recently, [7] has proposed a new multiuser model, called the Z-channel; see Figure 1. The Z-channel is a special case of the $X$-channel in that there is only one crossover link and as a consequence, the transmitter that does not have a crossover link has only one message to send. In [7], an achievable region for the Gaussian Z-channel is provided for the case of $\alpha>$ $1+P_{1}$. In this paper, we focus on the model of the Gaussian Zchannel where the crossover link is weak, more specifically, $\alpha<1$. We derive an achievable region and claim that this region is almost equal to the capacity region by proving most of the converse. We also derive some lower and upper bounds on the capacity region. Finally, for the special case of $\alpha=1$, we determine the capacity region exactly.

\section{SySTEM Model}

The Gaussian Z-channel has two transmitters and two receivers as shown in Figure 1. The received signals at receivers $\mathrm{R} 1$ and $\mathrm{R} 2$ are given as,

$$
\begin{aligned}
& Y_{1}=X_{1}+\sqrt{\alpha} X_{2}+Z_{1} \\
& Y_{2}=X_{2}+Z_{2}
\end{aligned}
$$

This work was supported by NSF Grants ANI 02-05330 and CCR 0311311; and ARL/CTA Grant DAAD 19-01-2-0011.

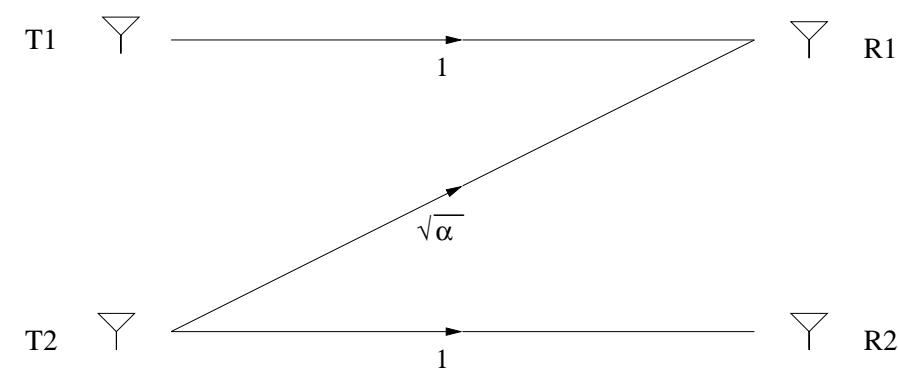

Fig. 1. The Z-channel.

where $X_{1}$ and $X_{2}$ are the signals transmitted by transmitters $\mathrm{T} 1$ and $\mathrm{T} 2$, and $Z_{1}, Z_{2}$ are independent Gaussian random variables with zero mean and unit variance. The transmitters T1 and T2 are subject to power constraints $P_{1}$ and $P_{2}$, respectively. The received signals in (1) and (2) can equivalently be written as,

$$
\begin{aligned}
Y_{1} & =\frac{X_{1}}{\sqrt{\alpha}}+X_{2}+\frac{Z_{1}}{\sqrt{\alpha}} \\
Y_{2} & =X_{2}+Z_{2}
\end{aligned}
$$

since scaling does not affect the capacity region. For the rest of this paper, we will be working with the channel model in (3) and (4).

Three independent messages are transmitted in a Z-channel: the message from transmitter $\mathrm{T} 1$ to receiver $\mathrm{R} 1$, denoted as $W_{11}$, the message from transmitter $\mathrm{T} 2$ to receiver $\mathrm{R} 1$, denoted as $W_{21}$, and the message from transmitter $\mathrm{T} 2$ to receiver $\mathrm{R} 2$, denoted as $W_{22}$. The capacity region of the Z-channel is a three dimensional volume, with axes $R_{11}, R_{21}$ and $R_{22}$ corresponding to the rates of messages $W_{11}, W_{21}$ and $W_{22}$.

In this paper, we mainly study the case of $\alpha<1$. Reference [7] studied the case of $\alpha>1+P_{1}$. These two cases correspond to two different kinds of "degradedness" conditions on the channels from transmitter T2 to both receivers. In the absence of the link between transmitter $\mathrm{T} 1$ and receiver $\mathrm{R} 1$, the channels from transmitter $\mathrm{T} 2$ to both receivers constitute a traditional BC [1]. Given the existence of the link from transmitter $\mathrm{T} 1$ to receiver $\mathrm{R} 1$, the condition, $\alpha>1+P_{1}$ assumed in [7], corresponds to the case that the signal of transmitter T2 received at receiver R2 is a "degraded" version of the same signal received at receiver R1 (for Gaussian inputs). The condition, $\alpha<1$, that we assume in this paper, corresponds to the case that the signal of transmitter $\mathrm{T} 2$ received at receiver R1 is a "degraded" version of the same signal at receiver R2. The "degradedness" condition we have here is stronger than the one in [7], in that, it is valid for all 
distributions of $X_{1}$.

In this paper, we consider only deterministic encoders, which incur no loss in performance [8]. All logarithms are defined with respect to base $e$.

\section{ACHIEVABLE REGION}

Let us define four quantities:

$$
\begin{aligned}
c_{11}(\beta) & =\frac{1}{2} \log \left(1+\frac{P_{1}}{\alpha \beta P_{2}+1}\right) \\
c_{21}(\beta) & =\frac{1}{2} \log \left(1+\frac{\alpha(1-\beta) P_{2}}{\alpha \beta P_{2}+1}\right) \\
c_{22}(\beta) & =\frac{1}{2} \log \left(1+\beta P_{2}\right) \\
c_{1}(\beta) & =\frac{1}{2} \log \left(1+\frac{P_{1}+\alpha(1-\beta) P_{2}}{\alpha \beta P_{2}+1}\right)
\end{aligned}
$$

The following theorem states an achievable region for the Gaussian Z-channel when $\alpha<1$.

Theorem 1 If $\alpha<1$, the following region is achievable in the Gaussian Z-channel:

$$
\begin{aligned}
R_{11} & \leq c_{11}(\beta) \\
R_{21} & \leq c_{21}(\beta) \\
R_{22} & \leq c_{22}(\beta) \\
R_{11}+R_{21} & \leq c_{1}(\beta)
\end{aligned}
$$

for any $0 \leq \beta \leq 1$.

Before providing the proof of Theorem 1, we show an example of the achievable region in Figure 2, where $P_{1}=1$, $P_{2}=5$ and $\alpha=0.5$. The boundary of the capacity region is traced as we change $\beta$ from 0 to 1 , and interpret inequalities in (9)-(12) as equalities. Each fixed $\beta$ determines a pentagon on a plane parallel to the $R_{11}-R_{21}$ plane as defined by inequalities (9), (10) and (12), and also a rate $R_{22}$ as defined by inequality (11). Therefore, the achievable region is a concatenation of pentagons of varying sizes along the $R_{22}$ axis.

Proof (Theorem 1): For simplicity, we will not present probability of error calculations, but rather, we will describe a scheme the transmitters and receivers may use to achieve the region given in (9) to (12).

Fix a $\beta$ between 0 and 1 , it suffices to show that the two rate triplets: $\left(R_{11}, R_{21}, R_{22}\right)=\left(c_{11}(\beta), c_{1}(\beta)-c_{11}(\beta), c_{22}(\beta)\right)$ and $\left(R_{11}, R_{21}, R_{22}\right)=\left(c_{1}(\beta)-c_{21}(\beta), c_{21}(\beta), c_{22}(\beta)\right)$ are achievable. This is because, if these two triplets are achievable, then all other points of the region can be achieved by the usual time-sharing technique.

First, we will show that $\left(R_{11}, R_{21}, R_{22}\right)=\left(c_{11}(\beta), c_{1}(\beta)-\right.$ $\left.c_{11}(\beta), c_{22}(\beta)\right)$ can be achieved. Transmitter T2 dedicates $\beta P_{2}$ power for transmitting message $W_{22}$ using codebook $C_{22}$, and $(1-\beta) P_{2}$ power for transmitting message $W_{21}$ using codebook $C_{21}$. It transmits the sum of the two codewords. Transmitter T1 uses all its power $P_{1}$ for transmitting message $W_{11}$ using codebook $C_{11}$.

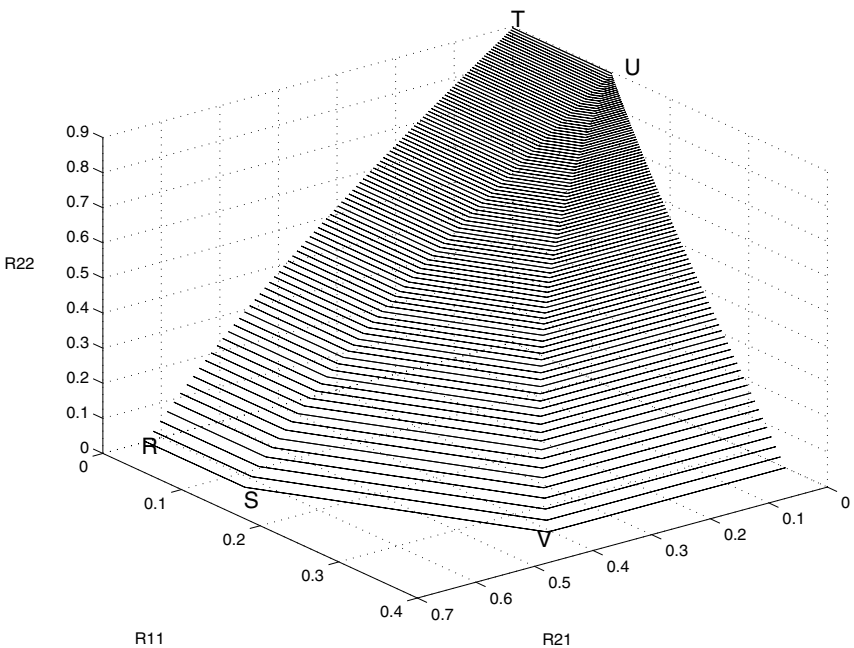

Fig. 2. The achievable region.

Receiver R1 looks at codebook $C_{21}$ only, treating everything else as noise, and therefore obtains a rate of

$$
R_{21}=\frac{1}{2} \log \left(1+\frac{(1-\beta) P_{2}}{\frac{P_{1}}{\alpha}+\beta P_{2}+\frac{1}{\alpha}}\right)=c_{1}(\beta)-c_{11}(\beta)
$$

Then, it subtracts the effect of $W_{21}$ off, looks at codebook $C_{11}$, treating everything else as noise, and obtains a rate of

$$
R_{11}=\frac{1}{2} \log \left(1+\frac{\frac{P_{1}}{\alpha}}{\beta P_{2}+\frac{1}{\alpha}}\right)=c_{11}(\beta)
$$

Together, this is a rate of $R_{11}+R_{21}=c_{1}(\beta)$.

Receiver R2, looks at codebook $C_{21}$ only, treating everything else as noise, since

$$
\begin{aligned}
R_{21} & =\frac{1}{2} \log \left(1+\frac{(1-\beta) P_{2}}{\frac{P_{1}}{\alpha}+\beta P_{2}+\frac{1}{\alpha}}\right) \\
& \leq \frac{1}{2} \log \left(1+\frac{(1-\beta) P_{2}}{\beta P_{2}+1}\right)
\end{aligned}
$$

it can decode $W_{21}$ without error. Subtracting the effect of $W_{21}$ off, looking at codebook $C_{22}$, receiver R2 gets a rate of

$$
R_{22}=\frac{1}{2} \log \left(1+\beta P_{2}\right)=c_{22}(\beta)
$$

Thus, rate triplet $\left(c_{11}(\beta), c_{1}(\beta)-c_{11}(\beta), c_{22}(\beta)\right)$ is achieved.

When both transmitters and receiver R2 operate in exactly the same way as explained above, and receiver R1 performs the successive decoding in the reverse order (i.e., it decodes $W_{11}$ first and then $\left.W_{21}\right)$, the rate triplet $\left(R_{11}, R_{21}, R_{22}\right)=$ $\left(c_{1}(\beta)-c_{21}(\beta), c_{21}(\beta), c_{22}(\beta)\right)$ is achieved.

We have established the achievability of the region defined by (9)-(12). Next, we will investigate the converse of this achievable region. We will show that, in most places, the achievable region is actually tight, i.e., it is equal to the capacity region of the channel. 


\section{The Converse}

Theorem 2 The achievable rate triplets $\left(R_{11}, R_{21}, R_{22}\right)$ have to satisfy

$$
\begin{aligned}
R_{21} & \leq c_{21}(\beta) \\
R_{22} & \leq c_{22}(\beta) \\
R_{11}+R_{21} & \leq c_{1}(\beta)
\end{aligned}
$$

for some $0 \leq \beta \leq 1$.

Referring back to Figure 2, this theorem states that, of the three surfaces that make up the achievability region, two of them, the surface defined by $T R S U$ and the surface defined by $U S V$, are actually tight.

Proof (Theorem 2): We will prove this by using ideas similar to El Gamal's alternative proof [9] to Bergmans' proof [10].

Since there is no cooperation between the two receivers, the capacity region of this channel depends on the joint distribution $p\left(y_{1}, y_{2} \mid x_{1}, x_{2}\right)$ only through the two marginals $p\left(y_{1} \mid x_{1}, x_{2}\right)$ and $p\left(y_{2} \mid x_{1}, x_{2}\right)$ [4]. Thus, we will concentrate on the following channel which will yield the same capacity region as our original channel (3)-(4),

$$
\begin{aligned}
& Y_{1}=\frac{X_{1}}{\sqrt{\alpha}}+Y_{2}+\tilde{Z} \\
& Y_{2}=X_{2}+Z_{2}
\end{aligned}
$$

where $\tilde{Z}$ and $Z_{2}$ are Gaussian random variables with zero mean and variance $\frac{1}{\alpha}-1$ and 1 , respectively. Let rate triplets $\left(R_{11}, R_{21}, R_{22}\right)$ be achievable. Then by Fano's inequality [1], there exists an $\epsilon_{n}$ such that

$$
\begin{aligned}
H\left(W_{22} \mid Y_{2}^{n}\right) & \leq n \epsilon_{n} \\
H\left(W_{21}, W_{11} \mid Y_{1}^{n}\right) & \leq n \epsilon_{n}
\end{aligned}
$$

and as $n \rightarrow \infty, \epsilon_{n} \rightarrow 0$.

We develop a series of bounds on $R_{22}$,

$$
\begin{aligned}
n R_{22} & =H\left(W_{22}\right) \\
& =H\left(W_{22} \mid Y_{2}^{n}\right)+I\left(W_{22} ; Y_{2}^{n}\right) \\
& \leq H\left(W_{22} \mid Y_{2}^{n}\right)+I\left(W_{22} ; Y_{2}^{n} \mid W_{21}\right) \\
& =H\left(W_{22} \mid Y_{2}^{n}\right)+h\left(Y_{2}^{n} \mid W_{21}\right)-h\left(Y_{2}^{n} \mid W_{21}, W_{22}\right) \\
& =H\left(W_{22} \mid Y_{2}^{n}\right)+h\left(Y_{2}^{n} \mid W_{21}\right)-h\left(Z_{2}^{n}\right) \\
& \leq n \epsilon_{n}+h\left(Y_{2}^{n} \mid W_{21}\right)-\frac{n}{2} \log (2 \pi e)
\end{aligned}
$$

where (27) is obtained from (26) using the independence of messages $W_{21}$ and $W_{22}$, (29) is obtained from (28) because we consider deterministic encoders, thus given $W_{21}$ and $W_{22}$, we know $X_{2}^{n}$, and therefore the only remaining randomness is in $Z_{2}^{n}$. Finally, (30) follows from (23) and the fact that $Z_{2}^{n}$ is an i.i.d. Gaussian sequence with unit variance.

Next, we develop a bound for $R_{21}$,

$$
\begin{aligned}
n R_{21} & =H\left(W_{21}\right) \\
& =H\left(W_{21} \mid Y_{1}^{n}\right)+I\left(W_{21} ; Y_{1}^{n}\right) \\
& \leq H\left(W_{11}, W_{21} \mid Y_{1}^{n}\right)+I\left(W_{21} ; Y_{1}^{n} \mid W_{11}\right)
\end{aligned}
$$

$$
\begin{aligned}
= & H\left(W_{11}, W_{21} \mid Y_{1}^{n}\right)+h\left(Y_{1}^{n} \mid W_{11}\right) \\
& -h\left(Y_{1}^{n} \mid W_{11}, W_{21}\right) \\
\leq & n \epsilon_{n}+h\left(Y_{1}^{n} \mid W_{11}\right)-h\left(Y_{1}^{n} \mid W_{11}, W_{21}\right) \\
\leq & n \epsilon_{n}+\frac{n}{2} \log (2 \pi e)\left(P_{2}+\frac{1}{\alpha}\right)-h\left(Y_{1}^{n} \mid W_{11}, W_{21}\right)
\end{aligned}
$$

Finally, we develop a bound for $R_{11}+R_{21}$,

$$
\begin{aligned}
n\left(R_{11}+R_{21}\right)= & H\left(W_{11}, W_{21}\right) \\
= & H\left(W_{11}, W_{21} \mid Y_{1}^{n}\right)+I\left(W_{11}, W_{21} ; Y_{1}^{n}\right) \\
= & H\left(W_{11}, W_{21} \mid Y_{1}^{n}\right)+h\left(Y_{1}^{n}\right) \\
& -h\left(Y_{1}^{n} \mid W_{11}, W_{21}\right) \\
\leq & n \epsilon_{n}+h\left(Y_{1}^{n}\right)-h\left(Y_{1}^{n} \mid W_{11}, W_{21}\right) \\
\leq & n \epsilon_{n}+\frac{n}{2} \log (2 \pi e)\left(\frac{P_{1}}{\alpha}+P_{2}+\frac{1}{\alpha}\right) \\
& -h\left(Y_{1}^{n} \mid W_{11}, W_{21}\right)
\end{aligned}
$$

where (36) and (41) follow from [11, Lemma 2].

Consider the following series of inequalities,

$$
\begin{aligned}
\frac{n}{2} \log (2 \pi e)\left(\frac{1}{\alpha}\right) & =h\left(Y_{1}^{n} \mid W_{11}, W_{21}, W_{22}\right) \\
& \leq h\left(Y_{1}^{n} \mid W_{11}, W_{21}\right) \\
& \leq h\left(Y_{1}^{n} \mid W_{11}\right) \\
& \leq \frac{n}{2} \log (2 \pi e)\left(P_{2}+\frac{1}{\alpha}\right)
\end{aligned}
$$

Thus, there exists a $\beta \in[0,1]$, such that

$$
h\left(Y_{1}^{n} \mid W_{11}, W_{21}\right)=\frac{n}{2} \log (2 \pi e)\left(\beta P_{2}+\frac{1}{\alpha}\right)
$$

From (36), (41) and (46), we see that there exists a $\beta \in[0,1]$ such that

$$
\begin{aligned}
n R_{21} & \leq n \epsilon_{n}+n c_{21}(\beta) \\
n\left(R_{11}+R_{21}\right) & \leq n \epsilon_{n}+n c_{1}(\beta)
\end{aligned}
$$

Finally, for $R_{22}$, we argue as follows,

$$
\begin{aligned}
h\left(Y_{1}^{n} \mid W_{11}, W_{21}\right) & =h\left(\frac{X_{1}^{n}}{\sqrt{\alpha}}+Y_{2}^{n}+\tilde{Z}^{n} \mid W_{11}, W_{21}\right) \\
& =h\left(Y_{2}^{n}+\tilde{Z}^{n} \mid W_{11}, W_{21}\right) \\
& =h\left(Y_{2}^{n}+\tilde{Z}^{n} \mid W_{21}\right)
\end{aligned}
$$

where (50) follows because $X_{1}^{n}$ is a deterministic function of $W_{11}$, and (51) follows because $Y_{2}^{n}$ and $\tilde{Z}^{n}$ are independent of $W_{11}$.

Now, let us consider $h\left(Y_{2}^{n}+\tilde{Z}^{n} \mid W_{21}\right)$. We know that

$$
h\left(\tilde{Z}^{n} \mid W_{21}\right)=h\left(\tilde{Z}^{n}\right)=\frac{n}{2} \log (2 \pi e)\left(\frac{1}{\alpha}-1\right)
$$

Applying entropy power inequality [10, Lemma II], we have

$$
h\left(Y_{2}^{n}+\tilde{Z}^{n} \mid W_{21}\right) \geq \frac{n}{2} \log (2 \pi e)\left(\frac{e^{\frac{2}{n} h\left(Y_{2}^{n} \mid W_{21}\right)}}{2 \pi e}+\frac{1}{\alpha}-1\right)
$$


Combining (53) with (46) and (51), we have

$$
h\left(Y_{2}^{n} \mid W_{21}\right) \leq \frac{n}{2} \log (2 \pi e)\left(\beta P_{2}+1\right)
$$

Thus, from (30), we have

$$
n R_{22} \leq n \epsilon_{n}+c_{22}(\beta)
$$

Since $\epsilon_{n} \rightarrow 0$ as $n \rightarrow \infty$, using (47), (48) and (55), we obtain the inequalites (18), (19) and (20), proving the theorem.

The converse that is missing is the part that describes $R_{11}$, when $R_{21}$ is so small that $R_{11}+R_{21}<c_{1}(\beta)$. This will be addressed in the discussion section next by developing some lower and upper bounds on the capacity region.

\section{DISCUSSION}

As stated above, combining Theorems 1 and 2, we see that the achievable region in Theorem 1 for $R_{21}, R_{22}, R_{11}+R_{21}$ is in fact tight. The only unsureness comes from $R_{11}$.

As mentioned in [7], the Z-channel includes the MAC, the $\mathrm{BC}$ and the Z-interference channel as special cases. By setting $\beta=0$ in the achievable region in Theorem 1 , we get

$$
\begin{aligned}
R_{11} & \leq \frac{1}{2} \log \left(1+P_{1}\right) \\
R_{21} & \leq \frac{1}{2} \log \left(1+\alpha P_{2}\right) \\
R_{11}+R_{21} & \leq \frac{1}{2} \log \left(1+P_{1}+\alpha P_{2}\right) \\
R_{22} & =0
\end{aligned}
$$

which is exactly the capacity region for the Gaussian MAC with link gains 1 and $\sqrt{\alpha}$, and noise variance 1 [1]. By setting $P_{1}=0$ in the achievable region in Theorem 1, we get

$$
\begin{aligned}
& R_{22} \leq \frac{1}{2} \log \left(1+\beta P_{2}\right) \\
& R_{21} \leq \frac{1}{2} \log \left(1+\frac{\alpha(1-\beta) P_{2}}{\alpha \beta P_{2}+1}\right) \\
& R_{11}=0
\end{aligned}
$$

which is exactly the capacity region for the Gaussian BC with channel gains 1 and $\sqrt{\alpha}$, and noise variance 1 [1]. By setting $R_{21}=0$ in the capacity region of the Gaussian Z-channel, we should get the capacity region of the Gaussian Z-interference channel [4], which is still an open problem.

\section{A. Sum Capacity of the Gaussian Z-channel}

Similar to the Z-interference channel case, the sum capacity of the Gaussian Z-channel is known for $\alpha<1$ based on the achievable region of Theorem 1 and the converse theorem, Theorem 2. The sum capacity is

$$
\max _{0 \leq \beta \leq 1} c_{22}(\beta)+c_{1}(\beta)
$$

It can be easily verified that when $\beta=1$, we attain the maximum and the sum capacity for the Gaussian Z-channel when $\alpha<1$ is

$$
\frac{1}{2} \log \left(\frac{\left(1+P_{2}\right)\left(1+P_{1}+\alpha P_{2}\right)}{1+\alpha P_{2}}\right)
$$

The sum capacity is attained at point $U$ in Figure 2.

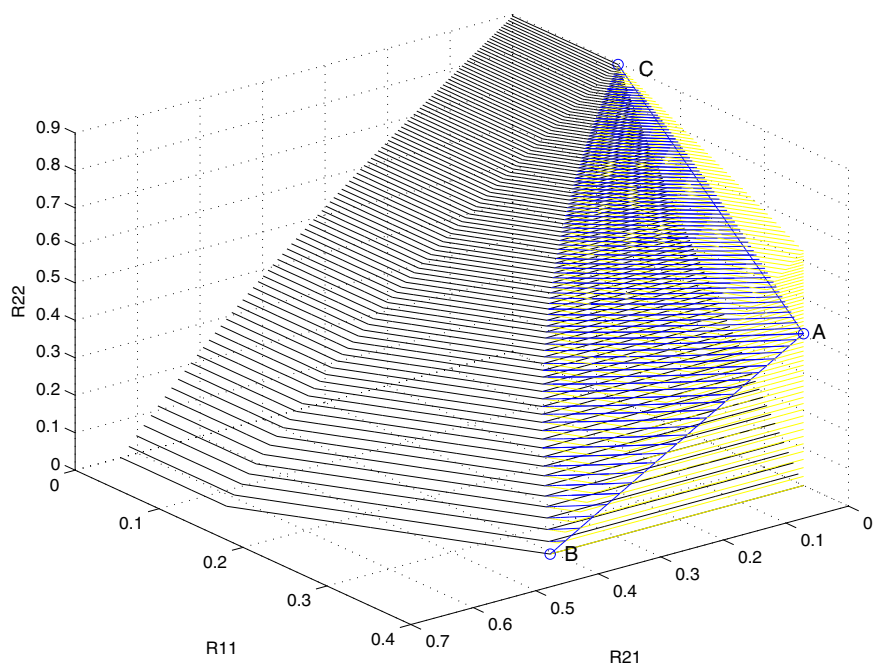

Fig. 3. All achievable regions and the upper bound in (65).

\section{B. Lower and Upper Bounds for the Capacity Region}

Next, we will derive lower and upper bounds for the capacity region for the portion where a converse is missing. An obvious upper bound for the capacity region is obtained by combining

$$
R_{11} \leq \frac{1}{2} \log \left(1+P_{1}\right)
$$

with (10), (11) and (12) for any $0 \leq \beta \leq 1$. In Figure 3, the achievable region in Theorem 1 is shown in black and this upper bound is shown in yellow.

Two other achievable regions can be derived to close the gap between the lower and upper bounds on the capacity region.

Larger Achievable Region 1: It is clear that the following three points given as triplets of $\left(R_{11}, R_{21}, R_{22}\right)$ are achievable.

$$
\begin{aligned}
& \text { Point } A:\left(\frac{1}{2} \log \left(1+P_{1}\right), 0, \frac{1}{2} \log \left(1+\frac{\alpha P_{2}}{P_{1}+1}\right)\right) \\
& \text { Point } B:\left(\frac{1}{2} \log \left(1+P_{1}\right), \frac{1}{2} \log \left(1+\frac{\alpha P_{2}}{P_{1}+1}\right), 0\right) \\
& \text { Point } C:\left(\frac{1}{2} \log \left(1+\frac{P_{1}}{\alpha P_{2}+1}\right), 0, \frac{1}{2} \log \left(1+P_{2}\right)\right)
\end{aligned}
$$

These three points are shown in Figure 3. Joining the lines between points $A$ and $B$ and points $A$ and $C$, and the curve connecting points $B$ and $C$, we can obtain a plane which is achievable by time sharing. It is also worth noting that the line $A B$ is optimal by the same argument as in [4, Theorem 1], i.e., line $A B$ is achievable and no point above line $A B$ on the plane of $R_{11}=\frac{1}{2} \log \left(1+P_{1}\right)$ is achievable.

Larger Achievable Region 2: Using the technique of succesive decoding [3], we can split $X_{2}$ into three parts:

$$
X_{2}=X_{21}+X_{c o m m}+X_{\text {priv }}
$$

where $X_{21}$ is a function of message $W_{21}$ and $X_{\text {comm }}+$ $X_{\text {priv }}$ together carry message $W_{22}$. Let $X_{\text {comm }}$ and $X_{\text {priv }}$ be independent. $X_{\text {comm }}$ is intended to be decoded by both 
receiver R1 and receiver R2, even though receiver R1 is not interested in decoding any part of message $W_{22} . X_{\text {priv }}$ is decoded by receiver R2 only. Receiver R1 treats $X_{\text {priv }}$ as noise. Transmitter T2 uses power $\bar{\gamma} P_{2}$ for $X_{21}$, power $\mu \gamma P_{2}$ for $X_{\text {comm }}$, and power $\bar{\mu} \gamma P_{2}$ for $X_{\text {priv }}$, where $\bar{\gamma}=1-\gamma$, $\bar{\mu}=1-\mu$ and $\gamma$ and $\mu$ vary from 0 to 1 .

Receiver R1 uses decoding order $X_{21}$, then $X_{\text {comm }}$ and finally $X_{1}$, and receiver R2 uses decoding order $X_{21}$, then $X_{\text {comm }}$ and finally $X_{\text {priv }}$. Let $\mathcal{A} 1(\mu, \gamma)$ be the set of $R_{11}$, $R_{21}$ and $R_{22}$ that satisfies the following inequalities:

$$
\begin{aligned}
R_{21} & \leq \frac{1}{2} \log \left(1+\frac{\bar{\gamma} P_{2}}{\frac{P_{1}}{\alpha}+\frac{1}{\alpha}+\gamma P_{2}}\right) \\
R_{\text {comm }} & \leq \frac{1}{2} \log \left(1+\frac{\mu \gamma P_{2}}{\frac{P_{1}}{\alpha}+\frac{1}{\alpha}+\bar{\mu} \gamma P_{2}}\right) \\
R_{11} & \leq \frac{1}{2} \log \left(1+\frac{\frac{P_{1}}{\alpha}}{\frac{1}{\alpha}+\bar{\mu} \gamma P_{2}}\right) \\
R_{\text {priv }} & \leq \frac{1}{2} \log \left(1+\bar{\mu} \gamma P_{2}\right) \\
R_{22} & =R_{\text {comm }}+R_{\text {priv }}
\end{aligned}
$$

Receiver R1 uses decoding order $X_{\text {comm }}$, then $X_{21}$ and finally $X_{1}$, and receiver R2 uses decoding order $X_{\text {comm }}$, then $X_{21}$ and finally $X_{\text {priv }}$. Let $\mathcal{A} 2(\mu, \gamma)$ be the set of $R_{11}, R_{21}$ and $R_{22}$ that satisfies the following inequalities:

$$
\begin{aligned}
R_{\text {comm }} & \leq \frac{1}{2} \log \left(1+\frac{\mu \gamma P_{2}}{\frac{P_{1}}{\alpha}+\frac{1}{\alpha}+\bar{\mu} \gamma P_{2}+\bar{\gamma} P_{2}}\right) \\
R_{21} & \leq \frac{1}{2} \log \left(1+\frac{\bar{\gamma} P_{2}}{\frac{P_{1}}{\alpha}+\frac{1}{\alpha}+\bar{\mu} \gamma P_{2}}\right) \\
R_{11} & \leq \frac{1}{2} \log \left(1+\frac{\frac{P_{1}}{\alpha}}{\frac{1}{\alpha}+\bar{\mu} \gamma P_{2}}\right) \\
R_{\text {priv }} & \leq \frac{1}{2} \log \left(1+\bar{\mu} \gamma P_{2}\right) \\
R_{22} & =R_{\text {comm }}+R_{\text {priv }}
\end{aligned}
$$

Then, an achievable region for the part where the converse is missing is the convex hull of

$$
\left(\bigcup_{0 \leq \mu, \gamma \leq 1} \mathcal{A} 1(\mu, \gamma)\right) \bigcup\left(\bigcup_{0 \leq \mu, \gamma \leq 1} \mathcal{A} 2(\mu, \gamma)\right)
$$

Figure 3 shows Larger Achievable Region 2 and the lines $A B$ and $A C$ defined in Larger Achievable Region 1 in blue. As we can see, there is still a gap between the lower and upper bounds, and additional research is needed to find the exact capacity region. We would like to mention here that using a coding scheme similar to [12], we would get an even larger achievable region than Larger Achievable Region 2.

\section{The Capacity Region when $\alpha=1$}

Finally, it is worth noting that the Gaussian Z-channel with $\alpha<1$ has the same capacity region as the channel in Figure 4 where $Z$ and $Z_{2}$ are zero-mean Gaussian random variables

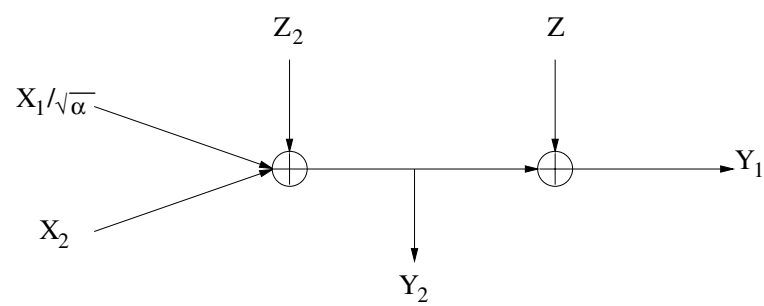

Fig. 4. The equivalent channel.

with variance $\frac{1}{\alpha}-1$ and 1 , respectively. This can be proved by the argument in [4, Appendix A]. Noting that the two channels have the same capacity is useful, since the capacity region of the channel in Figure 4 might be easier to determine in some cases. For example, for $\alpha=1, Y_{1}$ and $Y_{2}$ are statistically equivalent, thus both receiver $\mathrm{R} 1$ and receiver $\mathrm{R} 2$ are able to decode all three messages, $W_{11}, W_{12}$ and $W_{22}$, similar to a MAC. Thus, the capacity region of the Gaussian Z-channel with $\alpha=1$ is

$$
\begin{aligned}
R_{11} & \leq \frac{1}{2} \log \left(1+P_{1}\right) \\
R_{21}+R_{22} & \leq \frac{1}{2} \log \left(1+P_{2}\right) \\
R_{11}+R_{21}+R_{22} & \leq \frac{1}{2} \log \left(1+P_{1}+P_{2}\right)
\end{aligned}
$$

\section{CONCLUSION}

In this paper, we provided an achievable region for the recently proposed Gaussian Z-channel when $\alpha<1$. We were able to prove most of the converse for this achievable region. We also provided an upper bound and two larger achievable regions to characterize the capacity region better. We determined the exact capacity region when $\alpha=1$.

\section{REFERENCES}

[1] T. M. Cover and J. A. Thomas, Elements of Information Theory. WileyInterscience, 1991.

[2] E. van der Meulen, Some reflections on the interference channels. Kluwer Academic Publishers, 1994, pp. $409-421$.

[3] A. Carleial, "Interference channels," IEEE Trans. Inform. Theory, vol. 24, pp. $60-70$, Jan. 1978.

[4] M. Costa, "On the Gaussian interference channel," IEEE Trans. Inform. Theory, vol. 31, pp. $607-615$, Sep. 1985.

[5] G. Kramer, "Outer bounds on the capacity of Gaussian interference channels," IEEE Trans. Inform. Theory, vol. 50, pp. 581-586, Mar. 2004.

[6] I. Sason, "On acheivable rate regions for the Gaussian interference channel," IEEE Trans. Inform. Theory, vol. 50, pp. 1345-1356, Jun. 2004.

[7] S. Vishwanath, N. Jindal, and A. Goldsmith, "The "Z" channel," in IEEE Globecom, San Francisco, CA, Dec. 2003.

[8] F. Willems and E. van der Meulen, "The discrete memoryless multiple access channel with cribbing encoders," IEEE Trans. Inform. Theory, vol. 31, pp. $313-327$, May 1985.

[9] A. El Gamal, "EE478 Multiple User Information Theory," Fall 2002, Lecture notes.

[10] P. Bergmans, "A simple converse for broadcast channels with additive white Gaussian noise," IEEE Trans. Inform. Theory, vol. 20, pp. 279 -280 , Mar. 1974

[11] A. El Gamal, "The capacity of the physically degraded Gaussian broadcast channel with feedback," IEEE Trans. Inform. Theory, vol. 27, pp. 508-511, Jul. 1981.

[12] K. Marton, "A coding theorem for the discrete memoryless broadcast channel," IEEE Trans. Inform. Theory, vol. 25, pp. 306-311, May 1979. 